\title{
Some Asymptotic Results for the Number of Generalized Records
}

\author{
ANNE-LAURE FOUGÈRES \\ Laboratoire de Statistique et de Probabilités, Université Paul Sabatier, 118 Route de Narbonne, \\ F-31062, Toulouse Cedex 04, France \\ E-mail:fougeres@insa-toulouse.fr

\section{FABRICE GAMBOA} \\ Laboratoire de Statistique et de Probabilités, Université Paul Sabatier, 118 Route de Narbonne, \\ F-31062, Toulouse Cedex 04, France \\ E-mail: gamboa@math.ups-tlse.fr \\ CLÉMENTINE PRIEUR \\ Dépt GMM, Laboratoire de Statistique et Probabilités, INSA, 135 Avenue de Rangueil, F-31077, Toulouse \\ Cedex 04, France \\ E-mail:prieur@cict.fr
}

[Received 8 April 2004; Revised 7 March 2005; Accepted 18 May 2005]

\begin{abstract}
In this paper, we study asymptotic properties (large deviations and functional central limit theorem) of generalized record processes built on a triangular array of continuous and exchangeable random variables. As an application of these results, the links with the Kendall's rank correlation statistic are studied and testing exchangeability is discussed.
\end{abstract}

Key words. exchangeable distribution, generalized records, large deviations, rank

AMS 2000 Subject Classification.

\author{
Primary-60F 10 \\ Secondary-60F $17,62 \mathrm{G} 10$
}

\section{Introduction}

Let $X=\left(X_{k}^{(n)}\right)_{n \in \mathbb{N}^{*}, 1 \leq k \leq n}$ be a triangular array of exchangeable continuous random variables. That is, for any $n \in \mathbb{N}^{*}$, we assume that the distribution of the random vector $X^{(n)}=\left(X_{k}^{(n)}\right)_{k=1, \ldots, n}$ is invariant under any permutation of the indexes $\{1,2, \ldots, n\}$. In the particular case of a sequence of exchangeable random variables, that is when $X_{j}^{(n)}=$ $X_{j}$ for any $n \in \mathbb{N}^{*}$ and $j \in 1,2, \ldots, n$, de Finetti's theorem (Aldous, 1983) gives a representation formula for the distribution of $X^{(n)}\left(n \in \mathbb{N}^{*}\right)$. Indeed, it is well known that in this case the distribution of $X^{(n)}$ is that of a mixture of vectors of independent and identically distributed (i.i.d.) variables. In the whole paper we assume that, for $n \in \mathbb{N}^{*}$ and $j, j^{\prime} \in\{1, \cdots, n\}, j \neq j^{\prime}, X_{j}^{(n)} \neq X_{j^{\prime}}^{(n)}$ (a.s.). Following Resnick (1987) we define, for 
$n \in \mathbb{N}^{*}$ and $k \in\{1, \cdots, n\}$, the relative rank $R_{k}^{(n)}$ of $X_{k}^{(n)}$ as the number of coordinates in the random vector $\left(X_{j}^{(n)}\right)_{j=1, \ldots, k}$ greater than $X_{k}^{(n)}$ :

$$
R_{k}^{(n)}=\sum_{j=1}^{k} \mathbb{I}_{\left\{X_{j}^{(n)} \geq X_{k}^{(n)}\right\}} .
$$

Equivalently, if $X_{1, k}^{(n)} \leq \cdots \leq X_{k, k}^{(n)}$ are the order statistics of $X_{1}^{(n)}, \cdots, X_{k}^{(n)}$, the relative rank $R_{k}^{(n)}$ of $X_{k}^{(n)}$ can be defined as satisfying the relation $X_{k}^{(n)}=X_{k-R_{k}^{(n)}+1, k}^{(n)}$.

Using exchangeability, obvious combinatoric arguments lead to the following facts on the common distribution of the relative ranks (see Subsection 4.1 and Resnick (1987) for the i.i.d. case):

$$
\begin{aligned}
& \mathbb{P}\left(R_{k}^{(n)}=j\right)=\frac{1}{k}, \quad\left(n \in \mathbb{N}^{*}, k \in\{1, \cdots, n\}, j \in\{1, \cdots, k\}\right), \\
& R_{1}^{(n)}, \ldots, R_{n}^{(n)} \text { are independent random variables. }
\end{aligned}
$$

Notice that the distribution of $R_{1}^{(n)}, \ldots, R_{n}^{(n)}$ in our frame (exchangeable variables) is the same as in the case of i.i.d. variables. Therefore, any asymptotic result obtained on $\left(R_{j}^{(n)}\right)_{1<j<n, n \in \mathbb{N}}$ for an i.i.d. array $X$ still holds for an exchangeable array $X$. From now on, as for $k \in\{1, \cdots, n\}$ and $n \in \mathbb{N}^{*}$, the distribution of $\left(R_{1}^{(n)}, \ldots, R_{k}^{(n)}\right)$ only depends on $k$ we better write $R_{k}$ for $R_{k}^{(n)}$. Furthermore, we introduce the following processes, for $t \in$ $[0,1]$ :

$$
Z_{n}^{\alpha}(t)=\frac{\alpha}{n^{\alpha}} \sum_{j=1}^{\left[n t^{1 / \alpha}\right]} \mathbb{I}_{\left\{R_{j} \leq\left[j^{\alpha}\right]\right\}} \quad(0<\alpha<1)
$$

and

$$
Z_{n}^{0}(t)=\frac{1}{\log n} \sum_{j=1}^{\left[n^{t}\right]} \mathbb{I}_{\left\{R_{j}=1\right\}},
$$

where $[\cdot]$ denotes the integer part. The process $Z_{n}^{0}$ is the normalized number of usual records observed before $\left[n^{t}\right]$. Jumps in $Z_{n}^{0}$ locate the positions where a maximum value has been reached. Similarly, the process $Z_{n}^{\alpha}$ is the normalized number of the so-called "generalized records" observed before $\left[n t^{1 / \alpha}\right]$. Such generalized records have been considered by Deheuvels and Nevzorov (1994) in the i.i.d. setting only, but in a more general context, where the sequence $\left\{\left[j^{\alpha}\right], j \in \mathbb{N}^{*}\right\}$ is replaced by a sequence $\left\{k_{j}, j \in \mathbb{N}^{*}\right\}$. The last authors emphasized the motivation for the generalized records: Taking into account not only the records, but also a given proportion of "biggest observations" $k_{j}$, presents the benefit to waste less data, as more observations will take part of the process.

In this paper, we study some asymptotic results for these processes. Using a Poissonian approximation, we obtain a functional large deviations principle for $Z_{n}^{\alpha}$ $(0 \leq \alpha<1)$. The rate function is the same as the one associated to the large deviations for 
a standard Poisson process up to a factor $\alpha$. Furthermore, the same arguments show that Gaussian fluctuations hold for the centered and scaled processes:

$$
\tilde{Z}_{n}^{\alpha}(t)=\sqrt{\frac{n^{\alpha}}{\alpha}}\left\{Z_{n}^{\alpha}(t)-t\right\} \quad(0<\alpha<1, t \in[0,1])
$$

and

$$
\tilde{Z}_{n}^{0}(t)=\sqrt{\log n}\left\{Z_{n}^{0}(t)-t\right\}, \quad t \in[0,1] .
$$

Using this simple trick, we partially recover in a different way the general results on Gaussian approximations for the number of generalized records obtained in Deheuvels and Nevzorov (1994). Even if our proof happens to give these results only for the $\alpha<2 / 3$, it is an extension from the i.i.d. setting considered in Deheuvels and Nevzorov (1994) to the exchangeable setting.

The paper is organized as follows. The next section contains our asymptotic results for generalized record processes. To be selfcontained, we first recall some generalities on large deviations in Section 2.1. Then, we state a large deviations theorem (Theorem 2.3) and explain in a short proof how to build the Poissonian approximation. In Section 2.2 we give a functional central limit theorem for $\tilde{Z}_{n}^{\alpha}$. Section 3 is devoted to an application of our asymptotic results: The link with the Kendall's rank correlation statistic is exhibited, and the problem of testing randomness or exchangeability is discussed. All the technical proofs are postponed to the Appendix.

\section{Asymptotic results}

\subsection{Large deviations}

2.1.1. Some generalities on large deviations. Let us first recall what is a large deviation principle (LDP). For more on LDP we refer to Dembo and Zeitouni (1998). Let $\left(a_{n}\right)$ be an increasing positive sequence of real numbers going to infinity with $n$.

Definition 2.1: We say that a sequence $\left(Q_{n}\right)$ of probability measures on a measurable Hausdorff space $(U, \mathcal{B}(U))$ satisfies a LDP with speed $\left(a_{n}\right)$ and rate function $I$ if:

i) I is lower semicontinuous, with values in $\mathbb{R}^{+} \cup\{+\infty\}$.

ii) For any measurable set $A$ of $U$ :

$$
-I(\text { int } A) \leq \liminf _{n \rightarrow \infty} a_{n}^{-1} \log Q_{n}(A) \leq \limsup _{n \rightarrow \infty} a_{n}^{-1} \log Q_{n}(A) \leq-I(\operatorname{clo} A),
$$

where $I(A)=\inf _{\xi \in A} I(\xi)$ and $\operatorname{int} A$ (resp. clo $\left.A\right)$ is the interior (resp. the closure) of $A$.

We say that the rate function I is good if its level sets $\{x \in U: I(x) \leq a\}$ are compact for any $a \geq 0$. More generally, a sequence of $U$-valued random variables is said to satisfy a $L D P$ if their distributions satisfy a LDP. 
We will need the following well known large deviations techniques (see e.g., Dembo and Zeitouni (1998) chapter 4 p. 126 and 130).

- Contraction principle. Assume that $\left(Q_{n}\right)$ satisfies a LDP on $(U, \mathcal{B}(U))$ with good rate function $I$ and speed $\left(a_{n}\right)$. Let $T$ be a continuous mapping from $U$ to another space $V$. Then $Q_{n} \circ T^{-1}$ satisfies a LDP on $(V, \mathcal{B}(V))$ with speed $\left(a_{n}\right)$ and good rate function

$$
I^{\prime}(y)=\inf _{x: T(x)=y} I(x), \quad(y \in V) .
$$

- Exponential approximation. Assume that $U$ is metric and let $d$ denote the distance on $U$. Let $\xi=\left(\xi_{n}\right)$ and $\xi^{\prime}=\left(\xi_{n}^{\prime}\right)$ be two $U$-valued random sequences. If for any $\delta>0$,

$$
\limsup _{n \rightarrow \infty} a_{n}^{-1} \log \mathbb{P}\left(d\left(\xi_{n}, \xi_{n}^{\prime}\right)>\delta\right)=-\infty,
$$

we say that the sequences $\left(\xi_{n}\right)$ and $\left(\xi_{n}^{\prime}\right)$ are exponentially equivalent relatively to the sequence $\left(a_{n}\right)$. If $\xi$ satisfies a LDP with good rate function $I$ and speed $\left(a_{n}\right)$ and the sequences $\xi$ and $\xi^{\prime}$ are exponentially equivalent then $\xi^{\prime}$ shares the same LDP as $\xi$.

2.1.2. Main results. In this section we consider, for $\alpha \in[0,1]$, the process $\left\{Z_{n}^{\alpha}(t), t \in[0,1]\right\}$ as a random element of $L^{\infty}([0,1])$. This last space being equipped with the supremum norm. Let $\mathcal{A C}$ be the set of all absolutely continuous functions of $L^{\infty}([0,1])$ :

$$
\mathcal{A C}=\left\{\phi \in L^{\infty}([0,1]): \sum_{l=1}^{k}\left|t_{l}-s_{l}\right| \rightarrow 0, s_{l}<t_{l} \leq s_{l+1}<t_{l+1} \Rightarrow \sum_{l=1}^{k}\left|\phi\left(t_{l}\right)-\phi\left(s_{l}\right)\right| \rightarrow 0\right\} .
$$

Recall that any $\phi \in \mathcal{A C}$ is differentiable almost everywhere. We will denote by $\phi^{\prime}$ its derivative, which belongs to $L^{1}([0,1])$. Further, let $\mathcal{A C}_{0}^{+}$be the set of all non negative functions of $\mathcal{A C}$ vanishing at 0 . We now define an important functional in the study of large deviations for $\left\{Z_{n}^{\alpha}(t), t \in[0,1]\right\}$. For $\phi \in L^{\infty}([0,1])$, let

$$
J(\phi)=\left\{\begin{array}{cc}
\int_{0}^{1}\left[\phi^{\prime}(t) \log \phi^{\prime}(t)-\phi^{\prime}(t)+1\right] d t, & \text { if } \phi \in \mathcal{A C}_{0}^{+} \\
+\infty & \text { otherwise. }
\end{array}\right.
$$

Remark 2.2: Let $\left(N_{t}\right)_{t \geq 0}$ be the standard Poisson process. Then, for $T \rightarrow+\infty$ the process $\left\{T^{-1} N_{t T}, t \in[0,1]\right\}$ converges in probability to the deterministic process $\{t, t \in[0,1]\}$. Furthermore, this process satisfies a LDP in $\mathcal{A C}_{0}^{+}$with good rate function $J$ [see Léonard (2000)]. Moreover, one easily checks that for $\phi \in \mathcal{A C}_{0}^{+}, J(\phi)=0$ if and only if $\phi(t) \equiv t$.

One of our main results follows.

Theorem 2.3: For $0<\alpha<1($ resp. $\alpha=0),\left(Z_{n}^{\alpha}\right)$ satisfies in $L^{\infty}([0,1])$ a LDP with good rate function $J / \alpha($ resp. $J)$ and speed $\left(n^{\alpha}\right)($ resp. $(\log n))$. 
Proof: All our proofs rely on a simple Poissonian approximation argument. We will focus here on this approximation and postpone the remaining part of the proof to the Appendix. We first deal with the case $\alpha \neq 0$ and then give a sketch of the proof in the case $\alpha=0$.

First let

$$
Y_{j}^{\alpha}:=\mathbb{I}_{R_{j} \leq\left[j^{\alpha}\right]} \text { and } p_{j}^{\alpha}=\frac{\left[j^{\alpha}\right]}{j} \quad\left(j \in \mathbb{N}^{*}\right) .
$$

Obviously, from (1.2) and (1.3) the random variables $\left(Y_{j}^{\alpha}\right)$ are independent and, for $j \in \mathbb{N}^{*}, Y_{j}^{\alpha} \sim \mathcal{B}\left(p_{j}^{\alpha}\right)$ (Bernoulli distribution with parameter $p_{j}^{\alpha}$ ). We will now build from the standard Poisson process a sequence having the same distribution as the sequence $\left(Y_{j}^{\alpha}\right)$.

Recall that $\left(N_{t}\right)_{t \leq 0}$ is the standard Poisson process. Further, let $\left(N_{t}^{*}\right)_{t \geq 0}$ be defined by $N_{t}^{*}=N_{\frac{t^{\alpha}}{\alpha}}, t \geq 0$. Hence, $\left(N_{t}^{*}\right)_{t \geq 0}$ is the Poisson process with intensity function

$$
I(x)=x^{\alpha-1}, x>0 .
$$

Let

$$
Y_{j}^{\alpha \prime}=\mathbb{I}_{N_{j}^{*}-N_{j-1}^{*}>0}, j \in \mathbb{N}^{*} .
$$

Then $\left(Y_{j}^{\alpha \prime}\right)$ are independent and, for $j \in \mathbb{N}^{*}, Y_{j}^{\alpha \prime} \sim \mathcal{B}\left(p_{j}^{\alpha \prime}\right)$ with $p_{j}^{\alpha \prime}=1-e^{-\frac{1}{\alpha}\left(j^{\alpha}-(j-1)^{\alpha}\right)}$. Let now $W=\left(W_{j}\right)_{j \geq 1}$ and $W^{\prime}=\left(W_{j}^{\prime}\right)_{j \geq 1}$ be two sequences of independent random variables. Assume further that $W$ and $W^{\prime}$ are independent of the process $\left(N_{t}\right)_{t} \geq 0$ and have marginal distributions:

$$
W_{j} \sim \mathcal{B}\left(\min \left\{\frac{p_{j}^{\alpha}}{p_{j}^{\alpha \prime}}, 1\right\}\right), W_{j}^{\prime} \sim \mathcal{B}\left(\min \left\{\frac{1-p_{j}^{\alpha}}{1-p_{j}^{\alpha \prime}}, 1\right\}\right) .
$$

Observe that there is no loss of generality in supposing that for each $j$ one has

$$
Y_{j}^{\alpha}=W_{j} Y_{j}^{\alpha \prime} \mathbb{I}_{p_{j}^{\alpha} \leq p_{j}^{\alpha \prime}}+\left[1-W_{j}^{\prime}\left(1-Y_{j}^{\alpha \prime}\right)\right] \mathbb{I}_{p_{j}^{\alpha}>p_{j}^{\alpha \prime}}, j \in \mathbb{N}^{*},
$$

since this does not change the law of $Z_{n}^{\alpha}(\cdot)$. Now, using Lemma 4.1 the process $Z_{n}^{\alpha}(\cdot)$ is exponentially equivalent to the normalized process

$$
M_{n}^{\alpha}(t)=\frac{\alpha}{n^{\alpha}} N_{\left[n t^{1 / \alpha}\right]}^{*}, \quad t \in[0,1] .
$$

Therefore, we may conclude using Lemma 4.2.

As for the case $\alpha=0$, let consider $Y_{j}^{0}=\mathbb{I}_{R_{j}=1}, p_{j}^{0}=1 / j$ for $j \geq 2$ and $Y_{1}^{0}=p_{1}^{0}=1$. Here, we consider the Poisson process $N^{*}$ with intensity function $I(x)=1 / x$. Obviously, for $j \geq 2$, we have $\mathbb{P}\left(N_{j}^{*}-N_{j-1}^{*}>0\right)=1 / j$. Hence, as before, there is no loss of generality in supposing that for each $j \geq 2$ one has

$$
Y_{j}^{0}=\mathbb{I}_{N_{j}^{*}-N_{j-1}^{*}>0} .
$$


Making use of Lemma 4.1, one gets that the process $Z_{n}^{0}(\cdot)$ is exponentially equivalent to the normalized process

$$
M_{n}^{0}(t)=\frac{1}{\log n} N_{\left[n^{t}\right]}^{*}=\frac{1}{\log n} N_{[t \log n]}, \quad t \in[0,1],
$$

and conclude using Lemma 4.2.

\subsection{Donsker theorem}

The theorem stated in this section can be obtained in the i.i.d. case as a corollary of a general result by [see Deheuvels and Nevzorov (1994), Proposition 2.2] on strong approximation for record processes. However, we found it worthwile to reobtain it here, as our result is given here in the exchangeable case, and involves simple Poissonian approximation arguments, whereas Deheuvels \& Nevzorov's proof relies on Sakhanenko's (1985) results on strong approximation of partial sums of nonidentically distributed random variables.

As often for processes of Donsker's type, for $\alpha \in[0,1]$, the paths of $\left\{\tilde{Z}_{n}^{\alpha}(t), t \in[0,1]\right\}$ lie almost surely in the set of right continuous and left limited functions on [0,1] [càdlàg functions, see for example Billingsley (1999)].

Theorem 2.4: Let $\alpha \in\left[0, \frac{2}{3}\left[\right.\right.$. As $n$ goes to infinity, $\left\{\tilde{Z}_{n}^{\alpha}(t), t \in[0,1]\right\}$ converges in distribution to a standard Brownian motion on [0,1].

Proof: Similarly to the proof of Theorem 2.3, we will focus here on the main argument which relies once more on a simple Poissonian approximation. We postpone the rest of the proof to the Appendix. Moreover, in all the proofs, we deal with the case $\alpha \in] 0,1]$, as the proof for $\alpha=0$ follows exactly the same lines with another normalization and can be omitted. As in the proof of Theorem 2.3, one can assume that (2.2) holds. It is shown in Lemma 4.3 that for $\alpha \in\left[0, \frac{2}{3}\left[\right.\right.$, the process $\left\{\tilde{Z}_{n}^{\alpha}(t), t \in[0,1]\right\}$ can be approximated by the centered and rescaled process

$$
\tilde{M}_{n}^{\alpha}(t):=\sqrt{\frac{n^{\alpha}}{\alpha}}\left\{M_{n}^{\alpha}(t)-t\right\}=\sqrt{\frac{n^{\alpha}}{\alpha}}\left(\frac{\alpha}{n^{\alpha}} N_{\left[n t^{1 / \alpha}\right]}^{*}-t\right), \quad t \in[0,1],
$$

as one gets $\lim _{n \rightarrow \infty}\left\|\tilde{M}_{n}^{\alpha}-\tilde{Z}_{n}^{\alpha}\right\|=0$, where $\|\cdot\|$ denotes the classical supremum norm. We may then conclude using Lemma 4.4

Remark 2.5: As the Poissonian approximation argument is not valid for $\alpha \geq \frac{2}{3}$, we just write the Donsker Theorem for $\alpha \in\left[0, \frac{2}{3}[\right.$. However, the result has been proved for all $\alpha$ in $[0,1]$ by Deheuvels and Nevzorov, as mentioned previously. Their proof is given in the independent case, but could also be done in the same way in the exchangeable case. The condition $\alpha<2 / 3$ appearing in our assumption is an artefact of Poissonian approximation technique. Indeed, looking carefully at the proof of our central limit theorem, this condition is required when approximating Bernoulli random variables by Poisson ones [see (4.11)]. Thus, the Poissonian approximation tool is more convenient to study the 
large deviations properties of generalized records process than to get asymptotic normality properties of these processes.

Remark 2.6: Using the proof of Theorem 2.4 and the fact that a Poisson process has independent increments, one could check without major difficulties the following more general result: As $n$ goes to infinity, for $\alpha_{1}<\cdots<\alpha_{k} \in\left[0, \frac{2}{3}\left[,\left\{\left(\tilde{Z}_{n}^{\alpha_{1}}(t), \ldots, \tilde{Z}_{n}^{\alpha_{k}}(t)\right)\right.\right.\right.$, $t \in[0,1]\}$ converges in distribution to the standard Brownian motion in $\mathbb{R}^{k}$.

\section{Application: Testing randomness or exchangeability}

Given a time series $X_{1}, \cdots, X_{n}$ with a common continuous distribution function, a rather classical statistical problem consists of testing if the series can be seen as a white noise, i.e. if the observations are mutually independent or not. This hypothesis of randomness has been widely considered, and various statistics have been exploited to solve this problem. Wolfowitz (1943) showed that there does not exist any most powerful test against all possible alternatives. One of the main alternatives considered in the literature and the applications is the serial dependence. See for example Ferguson et al. (2000) or Genest et al. (2002) and references therein. Rank statistics are now well known to outperform correlogram techniques, as pointed out e.g., by Hallin and Mélard (1988), Hallin and Puri (1992) or Hallin and Werker (1999). One should mention that most of the test procedures for randomness actually rely on facts which are still true under exchangeability. This is for example the case with the classical test based on Kendall's rank correlation statistic [see e.g., Lehmann (1986)]

$$
\tau_{n}=1-\frac{4 N}{n(n-1)},
$$

where $N$ is the number of pairs in discordance defined by

$$
N=\sum_{i=1}^{n-1} \sum_{j=i+1}^{n} \mathbb{I}_{\left\{X_{i}>X_{j}\right\}}
$$

Observe that the Kendall's rank correlation statistic can be written in terms of the relative ranks defined by (1.1) via $N=\sum_{k=1}^{n} R_{k}-n$. Besides, straightforward calculus lead to the following result:

Lemma 3.1: If $X_{1}, \cdots, X_{n}$ comes from a triangular array $X=\left(X_{k}^{(n)}\right)$ of exchangeable and continuous random variables, the number of pairs in discordance defined by (3.1) and the relative ranks given by (1.1) satisfy the following, as $n$ tends to infinity:

$$
Z_{n}:=\frac{1}{n} \sum_{k=1}^{n} \frac{R_{k}}{k}-\frac{N}{n(n-1)} \stackrel{P}{\rightarrow} \frac{1}{4} .
$$

The proof easily follows from the fact that $\mathbb{E} Z_{n} \rightarrow 1 / 4$ and $\operatorname{Var} Z_{n} \rightarrow 0$. As a consequence, $\tau_{n}$ is asymptotically equivalent to $2-4 / n \sum_{k=1}^{n} R_{k} / k$. The classical test of 
randomness based on $\tau_{n}$ is therefore based on the mean behavior of the $R_{k} / k$ 's. One could besides base a test on a more local behavior of the $R_{k} / k$ 's, focusing on their tails. Such an approach is precisely provided by the statistic $n^{\alpha} / \alpha Z_{n}^{\alpha}(1)$ defined by (1.4). Critical regions are given by the Donsker theorem (see Theorem 2.4). One should notice that the complexity of the algorithm calculating the statistic $n^{\alpha} / \alpha Z_{n}^{\alpha}(1)$ is in $n^{2}$, as for Kendall's $\tau_{n}$. It seems realistic to argue against $n^{\alpha} / \alpha Z_{n}^{\alpha}(1)$ that it takes into account only part of the ranks information, whereas Kendall's statistic makes use of all this information. However, $\tau_{n}$ reflects the mean behavior of the $R_{k} / k$ 's, as mentioned above, whereas $n^{\alpha} / \alpha Z_{n}^{\alpha}(1)$ better describes their tail behavior. As a consequence, specific alternative hypotheses are likely to exist, which can be hard to detect via the mean behavior of the $R_{k} / k$ 's and more detectable by focusing on the tail. We were however not able so far to exhibit such kind of alternatives, and this remains an open problem.

\section{Appendix: Proofs}

\subsection{Proof of claims (1.2) and (1.3)}

Let $n>0$ and for $1 \leq j \leq n, r_{j} \in\{1,2, \ldots, j\}$. Following Resnick (1987) p. 169, there exists a unique permutation $\sigma$ on $\{1,2, \ldots, n\}$, depending on $\left(r_{j}\right)_{j}=1, \ldots, n$ such that:

$$
\left\{R_{1}^{(n)}=r_{1}, \ldots, R_{n}^{(n)}=r_{n}\right\}=\left\{X_{\sigma(1)}^{(n)}<X_{\sigma(2)}^{(n)}<\cdots<X_{\sigma(n)}^{(n)}\right\} .
$$

Furthermore, given a permutation $\sigma$ on $\{1,2, \ldots, n\}$ there exists a unique sequence $r_{j} \in$ $\{1,2, \ldots, j\}, j=1, \ldots, n$ satisfying (4.1). In other words, knowing relative ranks is equivalent to know how the sequence $\left(X_{j}^{(n)}\right)_{j}=1, \ldots, n$ is ordered. Now using exchangeability, for any permutation $\sigma$ on $\{1,2, \ldots, n\}$, we have

$$
\mathbb{P}\left(X_{\sigma(1)}^{(n)}<X_{\sigma(2)}^{(n)}<\cdots<X_{\sigma(n)}^{(n)}\right)=\mathbb{P}\left(X_{1}^{(n)}<X_{2}^{(n)}<\cdots<X_{n}^{(n)}\right) .
$$

We may thus deduce from the last equation that

$$
\mathbb{P}\left(R_{1}^{(n)}=r_{1}, \ldots, R_{n}^{(n)}=r_{n}\right)=\frac{1}{n !} .
$$

Hence, the random variables $R_{1}^{(n)}, \ldots, R_{n}^{(n)}$ are independent and, for $j=1, \ldots, n, R_{j}^{(n)}$ is uniformly distributed on $\{1,2, \ldots, j\}$. Notice that the previous proof is the same as the one given in Resnick (1987) for i.i.d. variables. Indeed, the crucial argument is (4.2), which remains valid in the exchangeable case.

\subsection{Lemmas for the proof of Theorem 2.3}

Lemma 4.1: For $\alpha \in[0,1]$ (resp. $\alpha=0$ ), the processes $\left\{Z_{n}^{\alpha}(t), t \in[0,1]\right\}$ and $\left\{M_{n}^{\alpha}(t)\right.$, $t \in[0,1]\}$ are exponentially equivalent, relatively to the sequence $\left(n^{\alpha}\right)($ resp. $(\log n))$. 
Proof: In the following we use the representations (2.2) in the case $0<\alpha<1$ or (2.3) in the case $\alpha=0$. Let, for $t \in[0,1]$

$$
Z_{n}^{\alpha \prime}(t)=\frac{\alpha}{n^{\alpha}} \sum_{j=1}^{\left[n t^{1 / \alpha}\right]} Y_{j}^{\alpha \prime}, A_{n}^{\alpha}(t)=\left|Z_{n}^{\alpha}(t)-Z_{n}^{\alpha \prime}(t)\right| \text { and } B_{n}^{\alpha}(t)=\left|Z_{n}^{\alpha \prime}(t)-M_{n}^{\alpha}(t)\right|
$$

We first begin with the case $0<\alpha<1$. Recall that for a bounded function $f$ on $[0,1],\|f\|$ denotes the classical supremum norm $\|f\|=\sup _{t[0,1]}|f(t)|$. On one hand

$$
\left\|A_{n}^{\alpha}\right\| \leq \frac{\alpha}{n^{\alpha}} \sum_{j=1}^{n}\left|Y_{j}^{\alpha}-Y_{j}^{\alpha \prime}\right|=: \frac{\alpha}{n^{\alpha}} \sum_{j=1}^{n} \chi_{j}^{\alpha}
$$

where $\left(\chi_{j}^{\alpha}\right)$ is a sequence of independent random variables with, for $j \in \mathbb{N}^{*}, \chi_{j}^{\alpha} \sim \mathcal{B}\left(\theta_{j}^{\alpha}\right)$ and $\theta_{j}^{\alpha}=\left|p_{j}^{\alpha}-p_{j}^{\alpha \prime}\right|$. Further, for $\tau \in \mathbb{R}$, using the independence,

$$
\begin{aligned}
\psi_{n}(\tau) & :=\frac{1}{n^{\alpha}} \log \mathbb{E}\left(e^{\tau \alpha \sum_{j=1}^{n} \chi_{j}^{\alpha}}\right)=\frac{1}{n^{\alpha}} \sum_{j=1}^{n} \log \mathbb{E}\left(e^{\tau \alpha \chi_{j}^{\alpha}}\right) \\
& =\frac{1}{n^{\alpha}} \sum_{j=1}^{n} \log \left(1-\theta_{j}^{\alpha}+\theta_{j}^{\alpha} e^{\alpha \tau}\right) .
\end{aligned}
$$

A Taylor expansion leads to $\theta_{j}^{\alpha}=\mathcal{O}\left(j^{\max (2 \alpha-2,-1)}\right)$ when $j$ goes to infinity, as

$$
\begin{aligned}
\theta_{j}^{\alpha} & =\left|p_{j}^{\alpha}-p_{j}^{\alpha \prime}\right|=\left|\frac{\left[j^{\alpha}\right]}{j}-1+\exp \left(-\frac{1}{\alpha} j^{\alpha}\left\{1-\left(1-\frac{1}{j}\right)^{\alpha}\right\}\right)\right| \\
& =\left|\frac{\left[j^{\alpha}\right]}{j}-j^{\alpha-1}+\frac{1}{2} j^{2 \alpha-2}+o\left(j^{2 \alpha-2}\right)\right|=\mathcal{O}\left(j^{-1}\right)+\mathcal{O}\left(j^{2 \alpha-2}\right) .
\end{aligned}
$$

For each $-1 / 2<x<1 / 2$, one has $x-x^{2} \leq \log (1+x) \leq x$. As a consequence, there exists an integer $j_{0}$ such that we get

$$
\frac{1}{n^{\alpha}} \sum_{j=j_{0}}^{n}\left\{K \theta_{j}^{\alpha}-\left(K \theta_{j}^{\alpha}\right)^{2}\right\} \leq \frac{1}{n^{\alpha}} \sum_{j=j_{0}}^{n} \log \left(1+K \theta_{j}^{\alpha}\right) \leq \frac{1}{n^{\alpha}} \sum_{j=j_{0}}^{n} K \theta_{j}^{\alpha}
$$

where $K=e^{\alpha \tau}-1$.

Let consider the term $1 / n^{\alpha} \sum_{j=1}^{n} K \theta_{j}^{\alpha}$; as $\theta_{j}^{\alpha}=\mathcal{O}\left(j^{\max (2 \alpha-2,-1)}\right)$, there exists a positive $C$ such that for all $j, 0<\theta_{j}^{\alpha} \leq C j^{\eta_{\alpha}}$, where $\eta_{\alpha}:=\max (2 \alpha-2,-1)$. Hence,

$$
\left|\frac{1}{n^{\alpha}} \sum_{j=1}^{n} K \theta_{j}^{\alpha}\right| \leq C|K| \frac{1}{n^{\alpha}}\left\{1+\sum_{j=2}^{n} j^{\eta_{\alpha}}\right\} \leq C|K| \frac{1}{n^{\alpha}}\left\{1+\int_{1}^{n} t^{\eta_{\alpha}} d t\right\} .
$$


It follows therefore from the last majoration that $1 / n^{\alpha} \sum_{j=1}^{n} K \theta_{j}^{\alpha}$ tends to 0 . One deduces easily from (4.3) and the above convergence that for any $\tau \in \mathbb{R}, \lim _{n \rightarrow \infty} \psi_{n}(\tau)=0$.

Using Markov exponential inequality, we obtain, for $\delta, \tau>0$

$$
n^{-\alpha} \log \mathbb{P}\left(\left\|A_{n}^{\alpha}\right\|>\delta\right) \leq \psi_{n}(\tau)-\tau \delta .
$$

Therefore, $\lim \sup _{n \rightarrow \infty} n^{-\alpha} \log \mathbb{P}\left(\left\|A_{n}^{\alpha}\right\|>\delta\right) \leq-\tau \delta$. As, $\tau>0$ is arbitrarily big we may conclude that $\left(Z_{n}^{\alpha}\right)$ and $\left(Z_{n}^{\alpha^{\prime}}\right)$ are exponentially equivalent.

On the other hand, as we always have $M_{n}^{\alpha}(t) \geq Z_{n}^{\alpha \prime}(t)$, we may write

$$
\begin{aligned}
\left\|B_{n}^{\alpha}\right\| & =\frac{\alpha}{n^{\alpha}} \sup _{t \in[0,1]} \sum_{j=1}^{\left[n t \frac{1}{\alpha}\right]}\left(N_{j}^{*}-N_{j-1}^{*}-\mathbb{I}_{N_{j}^{*}-N_{j-1}^{*}>0}\right) \\
& =\frac{\alpha}{n^{\alpha}} \sum_{j=1}^{n}\left(V_{j}^{\alpha}-1\right)_{+}, \quad \alpha \in[0,1],
\end{aligned}
$$

where $\left(V_{j}^{\alpha}\right)$ is a sequence of independent Poisson random variables with mean $\mu_{j}=-\log \left(1-p_{j}^{\alpha^{\prime}}\right)=\left\{j^{\alpha}-(j-1)^{\alpha}\right\} / \alpha$, for each $j \in \mathbb{N}^{*}$. Moreover, if $V$ is Poisson distributed with mean $\mu>0$, one has, for each $\tau \in \mathbb{R}$,

$$
\mathbb{E}\left(e^{\tau \alpha(V-1)_{+}}\right)=\sum_{k=1}^{\infty} e^{\tau \alpha(k-1)} e^{-\mu} \frac{\mu^{k}}{k !}+P[V=0]=e^{-\mu-\tau \alpha} \sum_{k=1}^{\infty} e^{\tau \alpha k} \frac{\mu^{k}}{k !}+e^{-\mu} .
$$

Therefore, one gets

$$
\begin{aligned}
\log \mathbb{E}\left(e^{\tau \alpha(V-1)_{+}}\right) & =\log \left(e^{-\mu}\left[e^{-\tau \alpha}\left\{e^{\mu e^{\tau \alpha}}-1\right\}+1\right]\right) \\
& =-\mu+\log \left(1+e^{-\tau \alpha}\left(e^{\mu e^{\tau \alpha}}-1\right)\right) .
\end{aligned}
$$

Hence, for $\tau \in \mathbb{R}$,

$$
\begin{aligned}
\varphi_{n}(\tau) & :=\frac{1}{n^{\alpha}} \log \mathbb{E}\left(e^{\tau n^{\alpha}\left\|B_{n}^{\alpha}\right\|}\right) \\
& =\frac{1}{n^{\alpha}} \sum_{j=1}^{n}\left\{-\mu_{j}+\log \left(1+e^{-\tau \alpha}\left(e^{\mu_{j} e^{\tau \alpha}}-1\right)\right)\right\} .
\end{aligned}
$$

A Taylor expansion, for $j$ going to infinity, leads to

$$
-\mu_{j}+\log \left(1+e^{-\tau \alpha}\left(e^{\mu_{j} e^{\tau \alpha}}-1\right)\right)=\mathcal{O}\left(j^{2 \alpha-2}\right) .
$$

Hence, we may conclude that, for all $\tau \in \mathbb{R}$ and as $n$ goes to infinity, $\varphi_{n}(\tau)$ goes to zero. Arguing as in (4.5) implies that $Z_{n}^{\alpha^{\prime}}$ and $M_{n}^{\alpha}$ are exponentially equivalent, as are also $Z_{n}^{\alpha}$ and $M_{n}^{\alpha}$.

In the case $\alpha=0$, first observe that $A_{n}^{0} \equiv 0$. Hence, we only study $\left(B_{n}^{0}\right)$. As in (4.6), we may write

$$
\left\|B_{n}^{0}\right\|=\frac{1}{\log n} \sum_{j=2}^{n}\left(V_{j}^{0}-1\right)+\frac{1}{\log n},
$$


where $\left(V_{j}^{0}\right)$ is a sequence of independent Poisson random variables with mean $\mu_{j}=$ $-\log (1-1 / j)$, for each $j \leq 2$. Hence, similarly to the case $\alpha \neq 0$ observing that here

$$
-\mu_{j}+\log \left(1+e^{-\tau \alpha}\left(e^{\mu_{j} e^{\tau \alpha}}-1\right)\right)=\mathcal{O}\left(j^{-2}\right)
$$

completes the proof.

Lemma 4.2: For $\alpha \in[0,1]$ (resp. $\alpha=0),\left\{M_{n}^{\alpha}(t), t \in[0,1]\right\}$ satisfies in $L^{\infty}([0,1])$ a $L D P$ with speed $\left(n^{\alpha}\right)$ (resp. $\left.(\log n)\right)$ and good rate function $J / \alpha$ (resp. J).

Proof: We focus on the case $0<\alpha<1$. The case $\alpha=0$ can be treated similarly. On one hand, for any unbounded increasing positive sequence $\left(a_{n}\right)$, the process $\left\{a_{n}^{-1} N_{a_{n} t}, t \in[0,1]\right\}$ satisfies a LDP with good rate function $J$ defined by (2.1) and speed $\left(a_{n}\right)$ (see for example Proposition 3.1. p. 101 in Léonard (2000) and references therein). Hence, $\left\{\alpha n^{-\alpha} N_{\alpha^{-1} n^{\alpha} t}\right.$, $t \in[0,1]\}$ satisfies a LDP with speed $(n \alpha)$ and good rate function $J / \alpha$.

On the other hand, we will now show that $\left(\alpha n^{-\alpha} N_{\alpha^{-1} n^{\alpha} t}\right)$ and $\left(M_{n}^{\alpha}(t)\right)$ are exponentially equivalent. Define for $t \in[0,1]$ and $0<\alpha<1$,

$$
C_{n}^{\alpha}(t)=\left|M_{n}^{\alpha}(t)-\frac{\alpha}{n^{\alpha}} N_{\alpha^{-1} n^{\alpha} t}\right|=\frac{\alpha}{n^{\alpha}} N_{\alpha^{-1} n^{\alpha} t}-M_{n}^{\alpha}(t),
$$

and let $t_{j}=n^{-\alpha} j^{\alpha}$ for $j=0, \ldots, n$. Obviously, as the Poisson process is non decreasing we have,

$$
\left\|C_{n}^{\alpha}\right\| \leq \sup _{j=1, \cdots, n}\left(\frac{\alpha}{n^{\alpha}} N_{\alpha^{-1} n^{\alpha} t_{j}}-M_{n}^{\alpha}\left(t_{j-1}\right)\right)=\frac{\alpha}{n^{\alpha}} \sup _{j=1, \cdots, n}\left(N_{\alpha^{-1} n^{\alpha} t_{j}}-N_{\alpha^{-1} n^{\alpha} t_{j-1}}\right) .
$$

Hence, using the same notation as in the proof of Lemma 4.1 from the last inequality we obtain, for $\delta>0$,

$$
\begin{aligned}
\mathbb{P}\left(\left\|C_{n}^{\alpha}\right\| \geq \delta\right) & \leq \mathbb{P}\left(\max _{j=1, \ldots, n} V_{j}^{\alpha} \geq \frac{\delta n^{\alpha}}{\alpha}\right) \\
& =1-\prod_{j=1}^{n}\left\{1-\mathbb{P}\left(V_{j}^{\alpha} \geq \frac{\delta n^{\alpha}}{\alpha}\right)\right\} .
\end{aligned}
$$

Recall that for $V \sim \mathcal{P}(\mu),(\mu>0)$ Chernoff exponential inequality gives (see for example Dacunha-Castelle and Duflo (1986) Chapter 2 Exercise 3).

$$
\mathbb{P}(V \geq(1+z) \mu) \leq \exp (-\mu H(z)) \text { with } H(z)=(1+z) \log (1+z)-z,(z>0)
$$

For $n$ large enough, so that $\delta n^{\alpha}>\alpha \mu_{j}$, we get therefore

$$
\mathbb{P}\left(\left\|C_{n}^{\alpha}\right\| \geq \delta\right) \leq 1-\prod_{j=1}^{n}\left(1-\exp \left\{-\mu_{j} H\left(\frac{\delta n^{\alpha}}{\alpha \mu_{j}}-1\right)\right\}\right)
$$


Besides, for such large enough $n$, one may write

$$
\begin{aligned}
& \prod_{j=1}^{n}\left[1-\exp \left\{-\mu_{j} H\left(\frac{\delta n^{\alpha}}{\alpha \mu_{j}}-1\right)\right\}\right] \\
& \geq \exp \left(n \min _{j=1, \cdots, n} \log \left[1-\exp \left\{-\mu_{j} H\left(\frac{\delta n^{\alpha}}{\alpha \mu_{j}}-1\right)\right\}\right]\right) .
\end{aligned}
$$

Note that the right part of the previous inequality is also equal to

$$
\exp \left(n \log \left[1-\exp \left\{-\min _{j=1, \cdots, n} \mu_{j} H\left(\frac{\delta n^{\alpha}}{\alpha \mu_{j}}-1\right)\right\}\right]\right)
$$

Besides, defining $\varphi(x)=\left\{x^{\alpha}-(x-1)^{\alpha}\right\} / \alpha H\left[\delta n^{\alpha} /\left\{x^{\alpha}-(x-1)^{\alpha}\right\}-1\right]$, one gets

$$
\min _{j=1, \cdots, n} \mu_{j} H\left(\frac{\delta n^{\alpha}}{\alpha \mu_{j}}-1\right) \geq \min \left\{\varphi(1), \min _{2 \leq x \leq n} \varphi(x)\right\} .
$$

Using the facts that $H$ is increasing on $\mathbb{R}_{+}$and that $x^{\alpha-1}<\left\{x^{\alpha}-(x-1)^{\alpha}\right\} / \alpha<$ $(x-1)^{\alpha-1}$ for each $x \in[2, n]$ and $\alpha<1$ then yields

$$
\varphi(x) \geq x^{\alpha-1} H\left(\frac{\delta n^{\alpha}}{\alpha(x-1)^{\alpha-1}}-1\right),(x \in[2, n]) .
$$

The right hand side of the inequality can be minored, replacing $H$ by its definition and using straightforward minoration, so that one gets, for each $x \in[2, n], 0<\alpha<1$ and $n$ large enough,

$$
\varphi(x) \geq \frac{\delta n^{\alpha}}{\alpha}\left\{\frac{1}{2^{1-\alpha}} \log \left(\delta n^{\alpha}\right)-1\right\} \geq \frac{\delta n^{\alpha} \log n}{2^{2-\alpha}} .
$$

On the other hand, one can obtain in a similar way that $\varphi(1)=H\left(\delta n^{\alpha}-1\right) / \alpha \geq$ $\delta n^{\alpha} \log n / 2$, and both inequalities lead to write finally, because of (4.10),

$$
1-\prod_{j=1}^{n}\left[1-\exp \left\{-\mu_{j} H\left(\frac{\delta n^{\alpha}}{\alpha \mu_{j}}-1\right)\right\}\right] \leq 1-\left\{\exp \left\{n \log \left(1-\exp \left\{\frac{-\delta n^{\alpha} \log n}{2^{2-\alpha}}\right\}\right)\right\}\right.
$$

Inequalities $1-e^{x} \leq-x \quad(x \in \mathbb{R})$ and $\log (1-x) \geq-2 x(0<x<1 / 2)$ yield then, for $n$ large enough,

$$
1-\prod_{j=1}^{n}\left[1-\exp \left\{-\mu_{j} H\left(\frac{\delta n^{\alpha}}{\alpha \mu_{j}}-1\right)\right\}\right] \leq 2 n \exp \left(-\frac{\delta n^{\alpha} \log n}{2^{2-\alpha}}\right)
$$

As a consequence, one gets from (4.9)

$$
\frac{1}{n^{\alpha}} \log \mathbb{P}\left(\left\|C_{n}^{\alpha}\right\| \geq \delta\right) \leq \frac{\log 2 n}{n^{\alpha}}-\frac{\delta}{2^{2-\alpha}} \log n
$$


Therefore

$$
\frac{1}{n^{\alpha}} \log \mathbb{P}\left(\left\|C_{n}^{\alpha}\right\| \geq \delta\right) \underset{[n \rightarrow+\infty]}{\longrightarrow}-\infty
$$

which concludes the proof of Lemma 4.2

\subsection{Lemmas for the proof of Theorem 2.4}

Lemma 4.3: For $\alpha \in\left[0, \frac{2}{3}\left[,\left\|\widetilde{Z}_{n}^{\alpha}-\widetilde{M}_{n}^{\alpha}\right\|\right.\right.$ tends to zero in probability.

Proof: Let, for $0<\alpha<1, t \in[0,1]$,

$$
\begin{aligned}
\tilde{Z}_{n}^{\alpha \prime}(t) & =\sqrt{\frac{n^{\alpha}}{\alpha}}\left(\frac{\alpha}{n^{\alpha}} \sum_{j=1}^{\left[n t^{1 / \alpha}\right]} Y_{j}^{\alpha \prime}-t\right), \tilde{A}_{n}^{\alpha}(t)=\left|\tilde{Z}_{n}^{\alpha}(t)-\tilde{Z}_{n}^{\alpha \prime}(t)\right| \text { and } \tilde{B}_{n}^{\alpha}(t) \\
& =\left|\tilde{Z}_{n}^{\alpha \prime}(t)-\tilde{M}_{n}^{\alpha}(t)\right| .
\end{aligned}
$$

Hence, it is sufficient to show that both $\left\|\tilde{A}_{n}^{\alpha}\right\|$ and $\left\|\tilde{B}_{n}^{\alpha}\right\|$ tend to zero in probability, for $\alpha \in[0,2 / 3[$. On one hand, keeping the notations of Section 4.2, one gets

$$
\left\|\tilde{A}_{n}^{\alpha}\right\| \leq \sqrt{\frac{\alpha}{n^{\alpha}}} \sum_{j=1}^{n}\left|Y_{j}^{\alpha}-Y_{j}^{\alpha \prime}\right|=\sqrt{\frac{\alpha}{n^{\alpha}}} \sum_{j=1}^{n} \chi_{j}^{\alpha} .
$$

Hence,

$$
\mathbb{E}\left\|\tilde{A}_{n}^{\alpha}\right\| \leq \sqrt{\frac{\alpha}{n^{\alpha}}} \sum_{j=1}^{n} \theta_{j}^{\alpha} .
$$

Then, as $\theta_{j}^{\alpha}=\mathcal{O}\left(j^{\max (2 \alpha-2,-1)}\right)$ as $j$ goes to infinity (see proof of Lemma 4.1), we easily check that $\left\|\tilde{A}_{n}^{\alpha}\right\|$ tends to zero in probability as soon as $\alpha<\frac{2}{3}$.

On the other hand, again keeping the notations of Section 4.2, we may write for each $\alpha \in[0,1]$

$$
\left\|\tilde{B}_{n}^{\alpha}\right\|=\sqrt{\frac{\alpha}{n^{\alpha}}} \sum_{j=1}^{n}\left(V_{j}^{\alpha}-1\right)_{+},
$$

where the $V_{j}^{\alpha}$ 's are independent Poisson random variables with mean $\mu_{j}=\left\{j^{\alpha}-\right.$ $\left.(j-1)^{\alpha}\right\} / \alpha$, for each $j \in \mathbb{N}^{*}$. As a consequence, using the easily checked fact that if $W$ is a Poisson random variable with mean $\lambda$, then $\mathbb{E}\left\{(W-1) \mathbb{I}_{W \geq 2}\right\}=\lambda+e^{-\lambda}-1$, we obtain

$$
\mathbb{E}\left\|\tilde{B}_{n}^{\alpha}\right\|=\sqrt{\frac{\alpha}{n^{\alpha}}} \sum_{j=1}^{n} \mathbb{E}\left(\left(V_{j}^{\alpha}-1\right) \mathbb{I}_{V_{j}^{\alpha} \geq 2}\right)=\sqrt{\frac{\alpha}{n^{\alpha}}} \sum_{j=1}^{n}\left\{\mu_{j}+e^{-\mu_{j}}-1\right\} .
$$


Replacing the $\mu_{j}$ 's by their definition yields, as $j$ tends to infinity, $\mu_{j}+e^{-\mu_{j}}-1=\mathcal{O}\left(j^{2 \alpha-2}\right)$. A similar argument as that used to get (4.4) leads to write,

$$
\mathbb{E}\left\|\tilde{B}_{n}^{\alpha}\right\|=\sqrt{\frac{\alpha}{n^{\alpha}}} \sum_{j=1}^{n} \mathbb{E}\left(\left(V_{j}^{\alpha}-1\right) \mathbb{I}_{V_{j}^{\alpha} \geq 2}\right)=\mathcal{O}\left(n^{3 \alpha / 2-1}\right),
$$

and therefore $\left\|\tilde{B}_{n}^{\alpha}\right\|$ tends to zero in probability as soon as $\alpha \in\left[0, \frac{2}{3}[\right.$.

Lemma 4.4: For $\alpha \in\left[0, \frac{2}{3}\left[,\left\{\tilde{M}_{n}^{\alpha}(t), t \in[0,1]\right\}\right.\right.$ converges in distribution to a standard Brownian motion on $[0,1]$.

Proof: We first recall Donsker Theorem for the standard Poisson process

$$
\left\{\frac{N_{t n^{\alpha} / \alpha}-t n^{\alpha} / \alpha}{\sqrt{n^{\alpha} / \alpha}}, t \in[0,1]\right\} \underset{[n \rightarrow+\infty]}{\stackrel{\mathcal{D}}{\longrightarrow}}\left\{W_{t}, t \in[0,1)\right\}
$$

where $\left\{W_{t}, t \in[0,1]\right\}$ denotes a standard Brownian motion on $[0,1]$ [see for example Billingsley (1999)]. For $t \in[0,1]$ and $0<\alpha<1$, let

$$
\tilde{C}_{n}^{\alpha}(t)=\left|\tilde{M}_{n}^{\alpha}(t)-\frac{N_{t n^{\alpha} / \alpha}-t n^{\alpha} / \alpha}{\sqrt{n^{\alpha} / \alpha}}\right|=\sqrt{\frac{\alpha}{n^{\alpha}}}\left(N_{t n^{\alpha} / \alpha}-N_{\left[t^{1 / \alpha} n\right]}^{*}\right) .
$$

In order to prove that $\left\{\tilde{M}_{n}^{\alpha}(t), t \in[0,1]\right\}$ converges in distribution to a standard Brownian motion on $[0,1]$, it is sufficient to show that $\left\|\tilde{C}_{n}^{\alpha}\right\|$ tends to zero in probability.

Recall that for $j=0, \ldots, n, t_{j}=n^{-\alpha} j^{\alpha}$. Obviously, as the Poisson process is non decreasing we have,

$$
\left\|\tilde{C}_{n}^{\alpha}\right\| \leq \sqrt{\frac{\alpha}{n^{\alpha}}} \sup _{j=1, \ldots, n}\left(N_{t_{j} n^{\alpha} / \alpha}-N_{\left[t_{j}^{1 / \alpha} n\right]}^{*}\right)
$$

Hence, using the same notations and calculus as in the proof of Lemma 4.2, we obtain from the last inequality and for $\delta>0$

$$
\mathbb{P}\left(\left\|\tilde{C}_{n}^{\alpha}\right\| \geq \delta\right) \leq \mathbb{P}\left(\max _{j=1, \ldots, n} V_{j}^{\alpha} \geq \delta \sqrt{\frac{n^{\alpha}}{\alpha}}\right)=1-\prod_{j=1}^{n}\left\{1-\mathbb{P}\left(V_{j}^{\alpha} \geq \delta \sqrt{\frac{n^{\alpha}}{\alpha}}\right)\right\}
$$

Then, replacing $n^{\alpha} / \alpha$ by $\sqrt{n^{\alpha} / \alpha}$ in inequality (4.9), we get for $n$ large enough

$$
\mathbb{P}\left(\left\|\tilde{C}_{n}^{\alpha}\right\| \geq \delta\right) \leq n \exp \left(\frac{-\delta \sqrt{\alpha}}{4} n^{\alpha / 2} \log n\right)
$$

As a consequence, $\left\|\tilde{C}_{n}^{\alpha}\right\|$ converges in probability to 0 , and $\left\{\tilde{M}_{n}^{\alpha}(t), t \in[0,1]\right\}$ converges to a standard Brownian motion. 


\section{Acknowledgments}

The authors warmly thank the referees who made very constructive comments, improving the writing and the proofs of the paper.

\section{References}

Aldous, D. Exchangeability and Related Topics. Lecture Notes in Math., 1117, Springer, Berlin, 1983.

Billingsley, P. Convergence of Probability Measures. Wiley series in probability and mathematical statistics, Wiley, New-York, 1999.

Dacunha-Castelle, D. and Duflo, M. Probability and Statistics, Vol. 1, Springer, New-York, 1986.

Deheuvels, P. and Nevzorov, V.B. "Limit laws for K-record times," J. Stat. Plan. Inference 38, 279-307, (1994). North-Holland.

Dembo, A. and Zeitouni, O. Large deviations techniques and applications. 2nd ed., Springer-Verlag, New York, 1998.

Ferguson, T.S., Genest, C. and Hallin, M. "Kendall's tau for serial dependence," Can. J. Stat. 28, 587-604, (2000).

Genest, C., Quessy, J.-F. and Rémillard, B. "Tests of serial independence based on Kendall's process," Can. J. Stat. 30, 1-21, (2002).

Hallin, M. and Mélard, G. "Rank-based tests for randomness against first-order serial dependence," J. Am. Stat. Assoc. 83, 1117-1128, (1988).

Hallin, M. and Puri, M., "Rank tests for time series analysis: A survey," in New Directions in Time Series Analysis, Part I (D.R. Brillinger, E. Parzen and M. Rosenblatt, eds.), Springer Verlag, New York, 111-153, (1992).

Hallin, M. and Werker, J.M., "Optimal testing for semi-parametric AR models_From Gaussian Lagrange multipliers to autoregression rank scores and adaptive tests." in Asymptotics, Nonparametrics, and Time Series (S. Ghosh, ed.), Marcel Dekker, New York, 295-350, (1999).

Lehmann, E.L. Testing Statistical Hypotheses, John Wiley \& Sons, New York, 1986.

Léonard, C. "Large deviations for Poisson random measures and processes with independent increments," Stoch. Process. their Appl. 85, 93-121, (2000).

Resnick, S.I. Extreme Values, Regular Variation, and Point Processes, Springer-Verlag, New York, 1987.

Sakhanenko, A.I. "Convergence rate in the invariance principle for non-identically distributed random variables with exponential moments," in Limit Theorems for Sums of Random Variables (A.A. Borovkov, ed.), Optimization Software Inc., New York, (1985).

Wolfowitz, J. "On the theory of runs with some applications to quality control," Ann. Math. Stat. 14, 280-288, (1943). 